\title{
Whole-body vibration improves neuromuscular parameters and functional capacity in osteopenic postmenopausal women
}

\author{
Milena C. Dutra, BS, ${ }^{1}$ Mônica L. de Oliveira, PhD, ${ }^{1}$ Rosangela V. Marin, MS, ${ }^{1}$ Hellen C.R. Kleine, BS, ${ }^{1}$ \\ Orivaldo L. Silva, $P h D,{ }^{2}$ and Marise Lazaretti-Castro, $P h D^{1}$
}

\begin{abstract}
Objective: In this longitudinal, paired-control study, we developed special vibration platforms to evaluate the effects of low-intensity vibration on neuromuscular function and functional capacity in osteopenic postmenopausal women.

Methods: Women in the platform group (PG; $\mathrm{n}=62)$ stood still and barefoot on the platform for 20 minutes, 5 times a week for 12 months. Each platform vibrated with a frequency of $60 \mathrm{~Hz}$, intensity of $0.6 \mathrm{~g}$, and amplitude of less than $1 \mathrm{~mm}$. Women in the control group $(C G ; n=60)$ were followed up and instructed not to modify their physical activity during the study. Every 3 months all volunteers were invited to a visit to check for any change in their lifestyle. Assessments were performed at baseline and at 12 months, and included isometric muscle strength in the hip flexors and back extensors, right handgrip strength, dynamic upper limb strength (arm curl test), upper trunk flexibility (reach test $[\mathrm{RT}]$ ), mobility (timed up and go test), and static balance (unipedal stance test). Statistical analyses were performed using the intention-to-treat strategy.

Results: Both groups were similar for all variables at baseline. At the end of intervention, the PG was significantly better than CG in all parameters but in the RT. When compared with baseline, after 12 months of vibration the PG presented statistically significant improvements in isometric and dynamic muscle strength in the hip flexors $(+36.7 \%)$, back extensors $(+36.5 \%)$, handgrip strength $(+4.4 \%)$, arm curl test $(+22.8 \%)$, RT $(+9.9 \%)$, unipedal stance test $(+6.8 \%)$, and timed up and go test $(-9.2 \%)$, whereas the CG showed no significant differences during the same period of time. As such, there were no side effects related to the study procedures during the 12 months of intervention.
\end{abstract}

Conclusions: Low-intensity vibration improved balance, motility, and muscle strength in the upper and lower limbs in postmenopausal women.

Key Words: Functional capacity - Muscle strength - Postmenopause - Sarcopenia and prevention Vibrating plate.

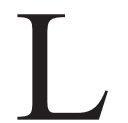
oss of muscle mass and strength, defined as sarcopenia, has been identified as a marker of health deterioration with aging. ${ }^{1}$ According to the literature, muscle strength decreases over the years, ${ }^{2}$ and a $5 \%$ decline in muscle mass is estimated to occur each decade after the age of 40 years, with a steeper decline after the age of 65 years. ${ }^{3}$

Specific guidelines recommend strength training to prevent sarcopenia and its consequences, bone mass loss, and osteoporotic fractures. Mechanical loading associated with resistance exercise induces musculoskeletal changes, stimulating

Received July 25, 2015; revised and accepted February 2, 2016.

From the ${ }^{1}$ Division of Endocrinology, Escola Paulista de Medicina, Universidade Federal de São Paulo, Sao Paulo, Brazil; and ${ }^{2}$ Department of Bioengeneering, Universidade de São Paulo (USP) de São Carlos, Sao Paulo, Brazil.

Funding/support: Grupo EDP (Energia de Portugal), Brazil.

Financial disclosure/conflicts of interest: None reported.

Address correspondence to: Marise Lazaretti-Castro, PhD, Division of Endocrinology, Escola Paulista de Medicina, Universidade Federal de São Paulo, Rua Bernarda Luis 68, CEP 05448-020, Sao Paulo, Brazil. E-mail: lazaretti.castro@unifesp.br local bone formation and remodeling (osteogenic muscle deformation). ${ }^{4}$

In the postmenopausal period, the decrease in sex hormones (estrogen, progesterone, and androgens) reduces the uptake of protein by the cells ${ }^{5}$ and changes the neurogenic stimulation of type II muscle fibers responsible for fast contraction, an important element in the proprioceptive reflexes. ${ }^{6}$ The most affected muscles are the knee extensors and hip flexors, important muscle groups involved in the maintenance of balance during gait. $^{7,8}$

Mechanical vibration delivered by vibrating platforms has been studied as a type of physical exercise to improve muscle strength and maintain bone mass. ${ }^{9,10}$ The effectiveness and the results of whole-body vibration depend on the nature, frequency, intensity of the stimulus, intensity of the vibration, type of acceleration (if vertical or oscillating), and type of population analyzed.

In the literature, studies with different designs have yielded different results. ${ }^{11-14}$ Most authors have included physical exercises on a vibrating platform to leverage the effects of muscle strength using vibration frequencies ranging from 20 
to $45 \mathrm{~Hz}$ and average intensities ranging from 2 to $5 \mathrm{~g},{ }^{15-18}$ where $1 \mathrm{~g}$ corresponds to the Earth's gravitational field $(9.8 \mathrm{~m} /$ $\mathrm{s}^{2}$ ). In contrast, the effects of vibrating platforms using lowvibration intensity $(<1 \mathrm{~g})$ and without associated physical exercise on neuromuscular parameters have been less studied. ${ }^{19,20}$ This has motivated us to develop and test a low-intensity vibrating platform specially designed for this study to verify the effects of mechanical vibration on neuromuscular parameters and functional capacity in postmenopausal women.

\section{METHODS}

The Ethics Committee of the Universidade Federal de São Paulo approved the study (protocol 0318/08), and all volunteers completed a free and informed consent form.

The platform, which was designed for this study by the Department of Bioengineering of the School of Engineering at USP São Carlos (patent protocol BR 202015010505 9Plataforma Vibratória para Tratamento e Prevenção da Osteoporose, filed on May 8, 2015), delivered vibration with a frequency of $60 \mathrm{~Hz}$, intensity of $0.6 \mathrm{~g}$, and amplitude of less than $1 \mathrm{~mm}$. The chosen vertical acceleration $(1.6 \mathrm{~g})$ mimics a moderate run $(\sim 10 \mathrm{~km} / \mathrm{h})$ and the frequency $(60 \mathrm{~Hz})$ mimics quite an intense race $(\sim 16.0 \mathrm{~km} / \mathrm{h})$, considering an average stride length $(\sim 1.2 \mathrm{~m})$.

The women were recruited through advertisements in the public media and events organized for this purpose. Women aged 55 or older with at least 5 years post menopause were included. Exclusion criteria were osteoporosis or another metabolic bone disease, use of medications that interfere with bone metabolism (except for calcium and vitamin D), recent history of bone fracture, osteoarthritis, primary hyperparathyroidism, hypothyroidism, rheumatoid arthritis, creatinine more than $1.4 \mathrm{mg} / \mathrm{dL}$, alcoholism, hypertension, poorly controlled diabetes, severe neuromuscular diseases, and chronic therapy with corticosteroids, bisphosphonates, estrogen, or selective estrogen-receptor modulators. Those participants available and committed to being present at the intervention site at the university 5 days a week for 12 months were selected for the platform group (PG). The control group (CG) was obtained from the same original population in a paired way.

\section{Design}

Women allocated to PG stood barefoot and still on a vibrating platform holding a support handle for 20 minutes, 5 days a week for 12 months under supervision (Fig. 1). There were 12 platforms available, so, for all women to be able to carry out their 20 minutes daily, about four daily shifts in different convenient periods of time were needed for the volunteers.

All women received instructions not to modify their physical activity during the study period. All participants from the control group as well as from the vibration group came every 3 months to check for any adverse events, to receive a health educational program as a retention plan, and to be sure that they did not modify their physical activity patterns. Adherence for the PG was measured by the frequency of vibration sessions, and those who had less than $80 \%$ were excluded. As for the CG, they were invited every 3 months to come to educational lectures. They were evaluated for possible changes in lifestyle or adverse events, and those who did not attend at least two calls or not performed the last evaluation were considered failures.

Women in both groups were evaluated with all tests and assessments. Measurement of serum vitamin D before the study showed optimal levels in all participants; therefore, supplementation was not required. We assessed the level of physical activity at baseline with the International Physical Activity Questionnaire-16. ${ }^{21}$ All other measurements were performed at baseline and at 12 months, and included bone density assessed by whole-body dual-energy x-ray absorptiometry (GE Lunar Model); upper trunk flexibility, measured with the reach test $(\mathrm{RT})^{22}$; isometric strength of the hip flexor and back extensor muscles determined with a portable mechanical dynamometer (Lafayette Manual Muscle Test System - Model 01163; Lafayette Instrument, IN ${ }^{23}$; right handgrip strength, assessed with the handgrip strength test using a Takei dynamometer ${ }^{24}$ (Grip - THE - Takei Physical Fitness Test - T.K.K. 5001, Japan); dynamic upper limb muscle
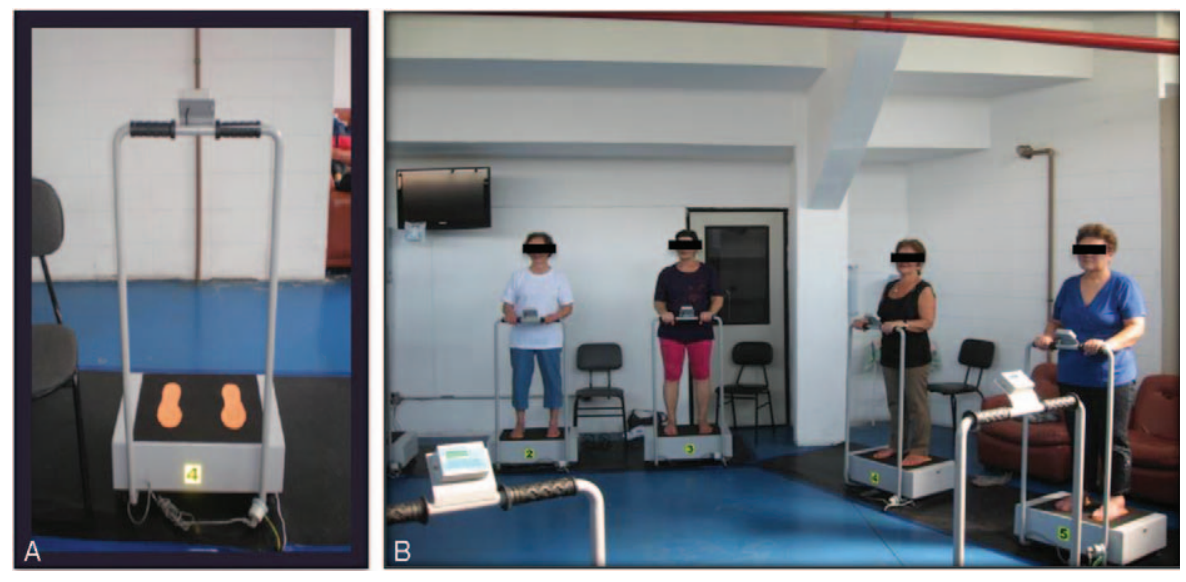

FIG. 1. Vibration platform's prototype (A) and the volunteers during a vibration session (B). 
TABLE 1. Comparison of all parameters from $P G$ and $C G$ groups at baseline and after 12 months

\begin{tabular}{|c|c|c|c|c|c|c|}
\hline Parameters & \multicolumn{2}{|c|}{ Baseline } & $P$ & \multicolumn{2}{|c|}{ After 12 mo } & $P$ \\
\hline Weight, $\mathrm{kg}$ & $63.8 \pm 12.0$ & $68.8 \pm 12.1$ & 0.042 & $62.8 \pm 12.1$ & $67.4 \pm 12.5$ & 0.121 \\
\hline Height, cm & $153.2 \pm 6.2$ & $153.1 \pm 6.0$ & 0.693 & $150.4 \pm 6.0$ & $153.2 \pm 5.8$ & 0.953 \\
\hline BMI & $27.4 \pm 3.9$ & $29.3 \pm 4.8$ & 0.563 & $27.0 \pm 4.2$ & $28.9 \pm 5.4$ & 0.069 \\
\hline Reach test, $\mathrm{cm}$ & $28.57 \pm 7.11$ & $28.95 \pm 8.64$ & 0.993 & $31.42 \pm 7.01$ & $28.23 \pm 6.88$ & 0.324 \\
\hline Hip flexor, $\mathrm{kg}$ & $10.45 \pm 4.54$ & $10.80 \pm 2.93$ & 0.735 & $14.22 \pm 5.95$ & $11.91 \pm 3.13$ & $<0.0001$ \\
\hline Back extensor, kg & $6.46 \pm 3.07$ & $6.30 \pm 1.79$ & 0.654 & $8.82 \pm 4.23$ & $7.19 \pm 2.16$ & $<0.0001$ \\
\hline Arm curl, rep & $15.06 \pm 2.28$ & $16.03 \pm 3.84$ & 0.161 & $18.50 \pm 4.24$ & $17.42 \pm 3.70$ & $<0.0001$ \\
\hline Handgrip, kg & $23.18 \pm 5.35$ & $22.96 \pm 3.54$ & 0.511 & $24.21 \pm 4.96$ & $22.74 \pm 4.60$ & $<0.0001$ \\
\hline
\end{tabular}

Results are presented as mean \pm standard deviations.

BMI, body mass index; CG, control group; PG, platform group; rep, repetitions.

strength, assessed with the elbow flexion by arm curl test ${ }^{25}$; mobility, determined with the timed up and go (TUG) test ${ }^{26}$; and static balance with eyes open, determined with the unipedal stance test. ${ }^{27}$ All tests were demonstrated to the women, and a tryout was conducted before their application. The participants performed the entire sequence of the tests after a voice command from an examiner.

\section{Statistical analysis}

The Kolmogorov-Smirnov test was used to confirm the normal distribution of the data. For comparisons of results with normal distribution at baseline and at 12 months, we used the Student's $t$ test for dependent variables and the Mann-Whitney $U$ test for nonparametric variables, performed as intention-to-treat strategy. The results are presented as means, SD, and delta percentages (\%). SPSS 16.0 for Windows was the statistical package used for all analyses. Results were considered significant when $P$ was $<0.05$.

\section{RESULTS}

Three hundred ninety-nine women filled out a questionnaire that contained the inclusion and exclusion criteria, and 264 were screened with bone densitometry. Of these, 122 who had osteopenia volunteered to participate in this longitudinal paired-control study and were divided into two groups: 62 women were selected for the PG based on their commitment to follow the vibration protocol and 60 others who agreed to participate as a control comprised the CG. Twenty-six women discontinued the study (10 from PG and 16 from CG) during the 12 months, so 96 women completed the study protocol. The reasons for discontinuation did not differ between groups, and included low compliance $(\mathrm{n}=15)$, family problems $(n=6)$, change in work shift $(n=1)$, use of a medication

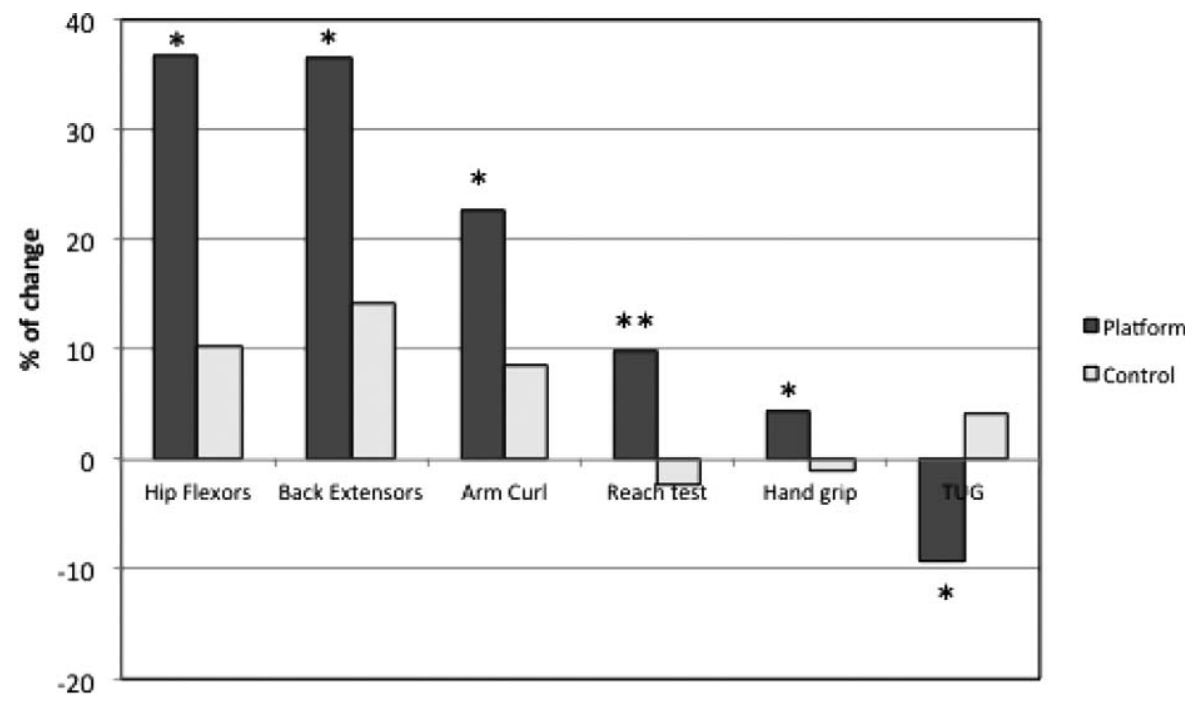

FIG. 2. Percentual changes in neuromuscular functions obtained in platform group after 12 months of a 20 -minute vibration per day for 5 days a week, and in control group. In the platform group all parameters showed a statistically significant improvement at the end of intervention compared with baseline and only the reach test was not different from the control group at the end. In the control group there were no significant changes in any parameter. TUG, timed up and go. ${ }^{*} P<0.05$ versus baseline and versus controls at 12 months; ${ }^{* *} P<0.05$ versus baseline. 
TABLE 2. Comparison of isometric and dynamic muscle strength parameters in the PG and CG groups at baseline and after 12 months of low-intensity vibration

\begin{tabular}{|c|c|c|c|c|c|c|c|c|}
\hline Isometric and dynamic muscle strength parameters & \multicolumn{4}{|c|}{ PG } & \multicolumn{4}{|c|}{ CG } \\
\hline Back extensor, $\mathrm{kg}$ & $6.46 \pm 3.07$ & $8.82 \pm 4.23$ & 36.5 & $<0.0001$ & $6.30 \pm 1.79$ & $7.19 \pm 2.16$ & 14.1 & 0.102 \\
\hline Arm curl, rep & $15.06 \pm 2.28$ & $18.50 \pm 4.24$ & 22.8 & $<0.0001$ & $16.03 \pm 3.84$ & $17.42 \pm 3.70$ & 8.6 & 0.232 \\
\hline Handgrip, kg & $23.18 \pm 5.35$ & $24.21 \pm 4.96$ & 4.4 & 0.036 & $22.96 \pm 3.54$ & $22.74 \pm 4.60$ & -0.9 & 0.972 \\
\hline
\end{tabular}

Results are presented as mean \pm standard deviations. Statistical analyses performed as intention to treat.

$\mathrm{CG}$, control group; PG, platform group; rep, repetitions.

considered an exclusion criterion for 3 months before the start of the study $(\mathrm{n}=1)$, health-related problems including surgery $(\mathrm{n}=1)$, acute pancreatitis $(\mathrm{n}=1)$, and heart problems $(\mathrm{n}=1)$. Apart from weight, both $\mathrm{PG}$ and $\mathrm{CG}$ had similar parameters at baseline, as shown in Table 1 .

After 12 months of the protocol, all neuromuscular parameters but the RT were significantly different between groups (Table 1), although the anthropometric measures were still similar. Although the CG had no significant changes in any parameter of isometric and dynamic muscle strength, in the PG all parameter significantly improved after 12 months of intervention (Fig. 2 and Table 2).

As for changes in functional capacity at 12 months, no significant differences in any variable were seen in the CG (Table 3). On the contrary, in PG there was a significant improvement of $9.9 \%$ in the flexibility assessed by RT and by $9.2 \%$ in the mobility, assessed by the TUG (Fig. 2 and Table 3). Only the unipedal stance test showed no differences.

\section{DISCUSSION}

This study aimed to evaluate the effects of low-intensity vibration delivered by a vibrating platform on postmenopausal women with low bone mass. Special platforms were developed specifically for the study using original technology. We demonstrated that low-intensity mechanical vibration produced by our platform improved neuromotor parameters and functional capacity in these women. Although other studies have reported effective results testing similar platforms, ${ }^{5,11,12,19}$ to our knowledge this was one of the few studies that demonstrated a gain in muscle strength as a result of low-intensity vibration without combined physical exercise. ${ }^{11,20}$

Muscle strength is a motor parameter that reduces physiologically with age, resulting in decreases in body mobility and subsequent limitations in daily activities in older individuals. ${ }^{8}$ In this study, the group of women allocated to vibration had, on average, a $36.7 \%$ increase in isometric strength in the hip flexors muscles. This muscle group is responsible for elevating the lower limb during the swing phase of gait, guaranteeing a good performance in body functionality, and reducing postural imbalance and risk of falls and fractures. ${ }^{28,29}$

Using a platform with low-level mechanical signals, Gilsanz et $\mathrm{al}^{11}$ observed using computed tomography a significant increase in muscle mass in the cross-sectional area of the psoas major muscle (5.2\% increase in the hip flexors muscle) and erector spine muscles $(7.2 \%$ increase in the postural muscles) when compared with a CG that did not undergo vibration. This study, conducted with young women (15-20 y), used a protocol with low-level vibration $(30 \mathrm{~Hz}, 0.3 \mathrm{~g})$ without combined physical exercise in which volunteers stood on the platform with extended knees for 10 minutes at a time over a period of 12 months.

Rubin et $\mathrm{al}^{30}$ demonstrated an attenuation of vibration along the body axis for frequencies higher than $25 \mathrm{~Hz}$. In their experiments, the transmissibility decreased to approximately $80 \%$ at the hip and spine in higher frequencies up to $35 \mathrm{~Hz}^{30}$ Nevertheless, one should consider that a $60 \mathrm{~Hz}$ platform as ours could transmit a sufficient amount of vibration to the hip and spine levels, which could explain our positive results at these sites. The act of standing on top of a low-frequency signal vibrating platform is sufficient to activate muscle motor units and induce muscle contractions. ${ }^{30,31}$ It has been demonstrated that the transmission range of low-frequency $(35-45 \mathrm{~Hz})$ and low-intensity $(0.3 \mathrm{~g})$ vibration can increase or decrease according to the position of the knees. ${ }^{11,29,32}$ When the knees are flexed, the vibration is delivered up to the lumbar-pelvic region, and when they are extended, the vibration can reach the entire spine. ${ }^{31}$ In our

TABLE 3. Comparison of functional capacity in the $P G$ and $C G$ groups at baseline and after 12 months of low-intensity vibration

\begin{tabular}{|c|c|c|c|c|c|c|c|c|}
\hline Functional capacity variables & \multicolumn{4}{|c|}{ PG } & \multicolumn{4}{|c|}{ CG } \\
\hline Unipedal stance test, s & $22.99 \pm 9.95$ & $24.56 \pm 8.49$ & 6.8 & 0.281 & $23.08 \pm 9.62$ & $26.44 \pm 6.97$ & 14.5 & 0.066 \\
\hline Timed up and go test, $\mathrm{s}$ & $7.01 \pm 2.09$ & $6.36 \pm 1.18$ & -9.2 & 0.002 & $7.08 \pm 1.59$ & $6.77 \pm 1.22$ & -4.3 & 0.683 \\
\hline
\end{tabular}

Results are presented as mean \pm standard deviations. Statistical analyses performed as intention to treat.

$\mathrm{CG}$, control group; $\mathrm{PG}$, platform group. 
study, we observed a $36.5 \%$ increase in spine extensors muscle strength in women in the PG group when compared with those in the $\mathrm{CG}$, suggesting that with extension of the knees the vibratory stimulus reaches the spine.

We also observed an increase in strength in the upper limbs demonstrated by increased isometric handgrip strength and in the dynamic strength of the elbow flexors by arm curl test. We hypothesize that the upper limbs may also have received the vibratory stimulus because the volunteers held on to a metal handle fixed to the platform (Fig. 1), which vibrated along with the platform.

The mechanisms for this apparent anabolic effect of vibration on muscles are not completely understood, but they resemble changes induced by the physical exercises. Vibration seems to stimulate receptors in muscle spindles by the deformation produced by the oscillatory displacement in the myotendinous junction of the muscle bundle. They are connected to the alpha motor neurons, capable of providing a muscle contraction particularly effective for fibers type I and II. This myotatic reflex generated by the low-intensity stimulation induces an increase in the expression of myosin in the muscle's sarcomere, thereby enhancing the performance of the contractions of the motor units. ${ }^{33,34}$ Furthermore, the vibratory stimulus causes an increase of peripheral blood flow by the shear forces on vessel walls, producing vasodilation and subsequent increase of muscle metabolism. ${ }^{35}$

The physical and biological mechanisms that control musculoskeletal adaptations are complex and involve interactions mediated by gravity, muscle contraction, and physical activity, as well as genetic and individual elements. ${ }^{36}$ The mechanical signals generated by vibrating platforms, even with low intensities $(<1 \mathrm{~g})$, however, seem to promote more intense stimuli to the musculoskeletal system than those promoted by daily activities. ${ }^{37,38}$ This may be important for activation of the muscles in participants with sarcopenia, who not only have loss of muscle strength and mass, but also have deterioration of type II muscle fibers (fast twitch), requiring greater neurogenic stimuli to promote more effective muscle contraction.

Our volunteers also showed significant improvement in mobility, as determined by the TUG test, decreasing the time of execution. This is a meaningful finding because impaired mobility is an important marker of fragility in older adults. ${ }^{39}$ This test represents a complex assessment of the overall conditioning of an individual because it evaluates the integration of the strength of the lower limbs and the dynamic balance. ${ }^{25}$ Significant improvements with vibration were also seen in parameters associated with flexibility, measured by the RT. According to evidence, vibration produces an increase in elasticity, possibly secondary to vasodilation induced by increased blood flow which may reduce the stiffness of the muscle fibers. ${ }^{40,41}$

The lack of a dummy PG is one of the more important limitations of this study, making it impossible to completely rule out the placebo effect in the treated group. The researchers could not design a "make-believe" placebo platform able to simulate the mechanical vibrations. The study design also had a difficulty to create a logistic strategy to blind the treatment, and this could negatively influence the behavior of the participants from CG (not PG). On the contrary, it is hard to believe that 20 minutes of standing up on the platform could strongly improve the neuromuscular function as observed, including with more objective measurements such as dynamometry, especially because the population was relatively young and were active postmenopausal women. Nevertheless, the modifications of their daily routine and the positive effects of socialization could play some role, and the study findings should be interpreted cautiously. To evaluate the impact of low-vibration intensity on functional capacity, we used a dynamometer and applied functional capacity tests. We found relevant results regarding strength gain and functional capacity, but we did not evaluate the physiological mechanisms involved in these processes.

It is important to point out that the vibration produced by the platforms, specially developed for this study, was safe because it has extremely low vertical displacement (measures showed that the amplitude of the vibration were in the micrometer range). So, just linear momentum is transmitted between the segments of the body, without displacements or impact between the articular surfaces. During the 12-month period of the study, there were no events of cramps, fatigue, thromboembolism, or any acute joint inflammation signs in PG volunteers.

Vibration platforms are nowadays very popular in fitness studios, and can reach very high intensity, up to $20 \mathrm{~g}$. These interventions with very large accelerations may expose many systems of the human body to significant health risks, including musculoskeletal and neural damage. To define the safety limits for individuals exposed to whole-body vibration and provide a benchmark on acceptable levels, a guideline was established by the International Organization for Standardization in 1978 (ISO 2631-1978). The ISO 2631 allows the application of acceleration greater than $1 \mathrm{~g}$ only for very short periods of time and in special conditions. Much attention has to be given to the intensity of vibration, specially taking into account the target population. In our case, osteopenic postmenopausal women already have an intrinsic higher risk for damage, so it is very important to know that even very low intensities $(<1 \mathrm{~g})$ can be effective to improve neuromuscular performance.

\section{CONCLUSIONS}

In conclusion, low-intensity mechanical vibration produced by vibrating platforms, specially developed for this study, was able to consistently improve different parameters of the neuromuscular function and functional capacity particularly related to muscle strength. This suggests that this type of platform can be a useful auxiliary tool for prevention of sarcopenia associated with aging. The improvements in balance, flexibility, and mobility observed in this study can contribute to reduce the risk of falls, physical trauma, and fractures in this high-risk population. Further studies should 
be developed to understand the mechanism of vibration on the neuromuscular system, and to evaluate whether the benefits of vibration may be replicated in other populations at risk for sarcopenia.

Acknowledgments: We thank Grupo EDP-ANEEL for the financial support to conduct the study; Unidade Mogiana de Diagnóstico por Imagem (UMDI) for conducting the bone densitometry tests; Universidade Brás Cubas de Mogi das Cruzes for hosting the project; Ms Maria Jovita Siqueira Vilella, project manager at EDP; the volunteers who agreed to participate in the study; and all individuals who collaborated in the project.

\section{REFERENCES}

1. Janssen I, Baumgartner RN, Ross R, Rosenberg IH, Roubenoff R. Skeletal muscle cutpoints associated with elevated physical disability risk in older men and women. Am J Epidemiol 2004;159:413-421.

2. Silva TAA, Frisoli Junior A, Pinheiro MM, Szejnfeld VL. Sarcopenia associada ao envelhecimento: aspectos etiológicos e opções terapêuticas [Sarcopenia and aging: etiological aspects and therapeutic options]. Rev Bras Reumatol 2006;46:391-397.

3. Tzankoff SP, Norris AH. Effect of muscle mass decrease on age-related BMR changes. J Appl Physiol Respir Environ Exerc Physiol 1977;43:1001-1006.

4. Hingorjo MR, Syed S, Qureshi MA. Role of exercise in osteoporosis prevention-current concepts. J Pak Med Assoc 2008;58:78-81.

5. Burger HG, Hale GE, Robertson DM, Dennerstein L. A review of hormonal changes during the menopausal transition: focus on findings from the Melbourne Women's Midlife Health Project. Hum Reprod Update 2007;13:559-565.

6. Cadore EL, Brentano MA, Lhullier FLR, Kruel LFM. Fatores relacionados com as respostas da testosterona e do cortisol ao treinamento de força [Factors concerned with the testosterone and cortisol response to strength training]. Rev Bras Med Esporte 2008;14:74-78.

7. Frontera WR, Hughes VA, Lutz KJ, Evans WJ. A cross-sectional study of muscle strength and mass in 45- to 78-yr-old men and women. $J$ Appl Physiol 1991;71:644-650.

8. Abellan van Kan G. Epidemiology and consequences of sarcopenia. $J$ Nutr Health Aging 2009;13:708-712.

9. Rubin C, Turner AS, Mallinckrodt C, Jerome C, McLeod K, Bain S. Mechanical strain, induced noninvasively in the high-frequency domain, is anabolic to cancellous bone, but not cortical bone. Bone 2002;30:445-452.

10. Oxlund BS, Ortoft G, Andreassen TT, Oxlund H. Low-intensity, highfrequency vibration appears to prevent the decrease in strength of the femur and tibia associated with ovariectomy of adult rats. Bone 2003;32:69-77.

11. Gilsanz V, Wren TA, Sanchez M, Dorey F, Judex S, Rubin C. Low-level, high-frequency mechanical signals enhance musculoskeletal development of young women with low BMD. $J$ Bone Miner Res 2006;21:1464-1474.

12. Robling AG, Turner $\mathrm{CH}$. Mechanotransduction in bone: genetic effects on mechanosensitivity in mice. Bone 2002;31:562-569.

13. Rubin CT, Sommerfeldt DW, Judex S, Qin Y. Inhibition of osteopenia by low magnitude, high-frequency mechanical stimuli. Drug Discov Today 2001;6:848-858.

14. Turner S, Torode M, Climstein M, et al. A randomized controlled trial of whole body vibration exposure on markers of bone turnover in postmenopausal women. J Osteoporos 2011;2011:710387.

15. Delecluse C, Roelants M, Diels R, Koninckx E, Verschueren S. Effects of whole body vibration training on muscle strength and sprint performance in sprint-trained athletes. Int J Sports Med 2005;26:662-668.

16. Rittweger J. Vibration as an exercise modality: how it may work, and what its potential might be. Eur J Appl Physiol 2010;108:877-904.

17. Voight M, Cook G. Poor neuromuscular control: training neuromuscular reactivation. In: Prentice WE, Voight ML, eds. Técnicas em Reabilitação Musculoesquelética [in Portuguese]. Porto Alegre: Artmed; 2003:91-118.

18. Verschueren SM, Roelants M, Delecluse C, Swinnen S, Vanderschueren $\mathrm{D}$, Boonen S. Effect of 6-month whole body vibration training on hip density, muscle strength, and postural control in postmenopausal women: a randomized controlled pilot study. J Bone Miner Res 2004;19:352-359.
19. Rubin C, Recker R, Cullen D, Ryaby J, McCabe J, McLeod K. Prevention of postmenopausal bone loss by a low-magnitude, high-frequency mechanical stimuli: a clinical trial assessing compliance, efficacy, and safety. $J$ Bone Miner Res 2004;19:343-351.

20. Gusi N, Raimundo A, Leal A. Low-frequency vibratory exercise reduces the risk of bone fracture more than walking: a randomized controlled trial. BMC Musculoskelet Disord 2006;7:92.

21. Pardini R, Matsudo SMM, Matsudo VKR, Araujo T, Andrade E, Braggion G. Validation of international physical activity questionnaire (IPAQ): pilot study in Brazilian young adults. Med Sci Sports Exerc 1997;29:S5-S9.

22. Duncan PW, Weiner DK, Chandler J, Studenski S. Functional reach: a new clinical measure of balance. J Gerontol 1990;45:M192-M197.

23. Martin HJ, Yule V, Syddall HE, Dennison EM, Cooper C, Aihie Sayer A. Is hand-held dynamometry useful for the measurement of quadriceps strength in older people? A comparison with the gold standard Bodex dynamometry. Gerontology 2006;52:154-159.

24. Matsudo SMM. Variáveis neuromotoras. In: Mali SA, ed. Avaliação do Idoso Fisica e Funcional, 3rd ed. SP, Brazil: Celafiscs; 2010. pp. 59-60.

25. Rikly RE, Jones CJ. Functional fitness normative scores for communityresiding older adults, ages 60-94. J Aging Phys Act 1990;7:162-181.

26. Podsiadlo D, Richardson S. The timed "Up \& Go": a test of basic functional mobility for frail elderly persons. J Am Geriatr Soc 1991;39:142-148.

27. Williams HG, Greene LS. Williams-Greene test of physical and motor function: laboratory report from the Motor Development/Motor Control Laboratory, Department of Exercise Science, University of South Carolina, Columbia. In: Spirduso WW, ed. Physical Dimensions of Aging. Champaign, IL: Human Kinetics; 1995.

28. Kawanabe K, Kawashima A, Sashimoto I, Takeda T, Sato Y, Iwamoto J. Effect of whole-body vibration exercise and muscle strengthening, balance, and walking exercises on walking ability in the elderly. Keio $J$ Med 2007;56:28-33.

29. Ribeiro GR, Domingues DO, Silva VA. Flexibility training and its relation with daily life activities in aging: a study review. Rev Bras Cienc Saude 2008;3:86-91.

30. Rubin C, Pope M, Fritton JC, Magnusson M, Hansson T, McLeod K. Transmissibility of 15 -hertz to 35 -hertz vibrations to the human hip and lumbar spine: determining the physiologic feasibility of delivering lowlevel anabolic mechanical stimuli to skeletal regions at greatest risk of fracture because of osteoporosis. Spine 2003;28:2621-2627.

31. Judex S, Rubin CT. Is bone formation induced by high-frequency mechanical signals modulated by muscle activity? J Musculoskelet Neuronal Interact 2010;10:3-11.

32. Pel JJ, Bagheri J, van Dam LM, et al. Platform accelerations of three different whole-body vibration devices and the transmission of vertical vibrations to the lower limbs. Med Eng Phys 2009;31:937-944.

33. Mettalach G, Polo-Parada L, Peca L, Rubin CT, Plattner F, Bibb JA. Enhancement of neuromuscular dynamics and strength behavior using extremely low magnitude mechanical signals in mice. $J$ Biomech 2014;47:162-167.

34. Burke D, Schiller HH. Discharge pattern of single motor units in the tonic vibration reflex of human triceps surae. J Neurol Neurosurg Psychiatry 1976;39:729-741.

35. Lohman B, Petrofsky J, Maloney-hinds C, Betts-schw H, Thorpe D. The effect of wole body vibration on lower extremity skin blood flow in normal subjects. Med Sci Monit 2006;13:71-76.

36. Judex S, Garman R, Squire M, Donahue LR, Rubin C. Genetically based influences on the site-specific regulation of trabecular and cortical bone morphology. J Bone Miner Res 2004;19:600-606.

37. Fritton SP, McLeod KJ, Rubin CT. Quantifying the strain history of bone: spatial uniformity and self-similarity of low-magnitude strains. J Biomech 2000;33:317-325.

38. Rosenberg IH. Sarcopenia: origins and clinical relevance. J Nutr 1997;127(5 suppl):990S-991S.

39. da Silva RG, Andreotti R, Gehring PR, et al. Effects of a whole body vibration training program on strength, power, and functional tests in the physically active elderly. Rev Bras Cineantropom Desempenho 2009; $11: 8$.

40. Lohman EB III, Petrofsky JS, Maloney-Hinds C, Betts-Schwab H, Thorpe D. The effect of whole body vibration on lower extremity skin blood flow in normal subjects. Med Sci Monit 2007;13:CR71-CR76.

41. Laughlin MH, Schrage WG. Effects of muscle contraction on skeletal muscle blood flow: when is there a muscle pump? Med Sci Sports Exerc 1999;31:1027-1035. 